\title{
Minimum Upper-Bound Filter of Markovian Jump Linear Systems with Generalized Unknown Disturbances *
}

\author{
Yuemei Qin ${ }^{\mathrm{a}, \mathrm{b}}$, Yan Liang ${ }^{\mathrm{a}, \mathrm{b}}$, Yanbo Yang ${ }^{\mathrm{a}, \mathrm{b}}$, Quan Pan ${ }^{\mathrm{a}, \mathrm{b}}$, Feng Yang ${ }^{\mathrm{a}, \mathrm{b}}$ \\ ${ }^{a}$ School of Automation, Northwestern Polytechnical University, Xi'an, P.R. China \\ ${ }^{\mathrm{b}}$ Key Laboratory of Information Fusion Technology, Ministry of Education, P.R. China
}

\begin{abstract}
This paper presents the estimation problem of Markovian jump linear systems (MJLSs) with generalized unknown disturbances (GUDs). There exist multiple uncertainties including Markovian switching parameters and GUDs, along with traditional random noises. Here, the state transition of MJLS is treated as the jump from one vertex to another on a fixed polyhedron whose vertex represents a mode. Since the transition is dependent on stochastic Markovian switching parameter, a more general polytopic system with stochastic weights is considered and the corresponding upper-bound filter (UBF) is derived. Then, the MJLS with GUDs is transformed into a special case of the considered polytopic system by letting the corresponding stochastic weight as the binary value constructed by Markovian switching parameters and hence the recursive UBF is obtained. The parameters in the derived UBF are further optimized in pursuit of the minimum upper bounds of estimation error covariances. The simulation via maneuvering target tracking shows the effectiveness of the proposed filter.
\end{abstract}

Key words: Markovian jump linear systems; upper-bound filter; unknown disturbances; polytopic systems; target tracking.

\section{Introduction}

Markovian jump linear systems (MJLSs) have wide and successful applications in many fields, for example, target tracking $[1,2]$, fault-tolerant control [3,4], process control [5], and signal processing [6]. In general, the estimation issue concerning MJLSs is in the framework of the multiple model (MM) and there are three generations of MM estimators with the last two generations for the MJLSs [2,7]. However, all these MM methods need Gaussian assumptions on both process noises and measurement noises. In some situations, such Gaussian assumption does not always hold. For example, in maneuvering target tracking, the significant attitude changes bring out significant variations of radar reflections, leading to high-tailed and non-Gaussian (also called "glint") measurement noises [8]. Meanwhile, the recursive calculation of the first two moments of the interested vec-

\footnotetext{
* This paper was not presented at any IFAC meeting. Corresponding author Y. Liang. Tel. 86-13572972504. Fax 8629-88431306.

Email addresses: qinyuemei@mail.nwpu.edu.cn (Yuemei Qin), liangyan@nwpu.edu.cn (Yan Liang), yangyanbo9@mail.nwpu.edu.cn (Yanbo Yang), quanpan@nwpu.edu.cn (Quan Pan), yangfeng@nwpu.edu.cn (Feng Yang).
}

tor sometimes is enough in practice. For instance, target tracking focuses on estimating the state including position and velocity (the first moment) and covariance (the second moment). In other words, calculating the conditional state distribution given measurements is not only too computation-intensive but also may not be necessary in the view of desirable balance between estimation accuracy and computation burden. Thus, it motivates the development of linear minimum-mean-square-error (LMMSE) estimator for the MJLS.

In [9], the LMMSE estimator for the MJLS was derived from geometric augment based on directly estimating $x_{k} \mathbf{1}_{\left\{\Theta_{k}=i\right\}}$ instead of the state $x_{k}$, where $\mathbf{1}_{\left\{\Theta_{k}=i\right\}}$ is an indicator being 1 if $\Theta_{k}=i$ or 0 otherwise; $\Theta_{k}$ is the state of Markov chain. And its error covariance can converge to the unique positive-semidefinite solution of an $\mathrm{Nn}$ dimensional algebraic Riccati equation under the conditions of mean square stability of the MJLS and the ergodicity of the associated Markov chain, where $n$ is the dimension of the state vector and $N$ is the number of states of the Markov chain. Furthermore, a time-invariant (a fixed-gain matrix) LMMSE estimator was derived for MJLSs [10]. By the fact that roundoff errors in solving the above Riccati equation may cause the loss of the symmetry and positive-semidefinition, an array implementation with the better numerical robustness was devel- 
oped [11]. Recently, the LMMSE estimations of MJLSs are also extended to the cases of stochastic coefficient matrices [12] and randomly delayed measurements [13].

However, all these methods for the MJLSs, including the MM methods and the LMMSE methods, never consider the presence of unknown disturbances (UDs). In fact, the UDs exist in many actual applications [14]. In target tracking, the sensor bias and deception jamming existing in sensor measurements can be modeled as the UDs to the nominal model $[14,15]$. In process control, the faults or failures can also be represented by UDs to the fault-free model in fault detection and isolation $[3,4]$.

By the fact that many actual applications in the complex environment always face multiple unpredictable disturbances/uncertainties, this paper formulates the estimation problem of MJLSs with generalized unknown disturbances (GUDs) in measurements. Here, the upperbound filter (UBF) for the more general polytopic system with stochastic weights (regarded as stochastic parameters) and GUDs is first derived by constructing the upper bounds of covariances of estimation errors. Then, the MJLS with GUDs is transformed into a special case of above polytopic system via parameter substitution and the UBF is obtained recursively through calculating the correlated relationship about Markovian switching parameters. Furthermore, the optimal parameters are derived in pursuit of the minimum upper bounds. The simulation about the maneuvering target tracking validates the proposed filter.

Throughout this paper, $I$ and $O$ are the identity matrix and zero matrix with proper dimensions, respectively. $(\cdot)$ denotes the same content as that in the previous parenthesis and $[\cdot]_{i, j}$ represents the $(i, j)$ th sub-block of the corresponding matrix. $E(\cdot)$ and 'col' represent the mathematical expectation operator and column vector, respectively. For any two square matrices $A$ and $B$, $A \geq B(A>B)$ means $A-B$ is positive semi-definite (positive definite). The symbol ' $:=$ ' means definition and ' $\otimes$ ' refers to the Kronecher product. An indicator function $\mathbf{1}_{\left\{\Theta_{k}=j\right\}}$ will be 1 if $\Theta_{k}=j$ or 0 otherwise.

The rest of this paper is organized as follows. The problem formulation is presented in Section 2. The UBF and the MUBF are given in Section 3. A simulation about maneuvering target tracking is presented in Section 4 to testify the proposed method. The conclusion is finally made. All proofs are presented in the Appendix.

\section{Problem formulation}

In maneuvering target tracking in the electronic countermeasures (ECMs) environment, the target maneuvering motion is usually described by Markovian switching of multiple models [2] while the sensor bias, deception jamming and linearization approximation lead to the time-varying GUDs in measurements [14]. In other words, there coexist the Markovian switching parameter and GUDs, which motivates us to formulate the following discrete-time MJLS with GUDs in measurements:

$$
\begin{gathered}
x_{k+1}=F_{\Theta_{k}} x_{k}+G_{\Theta_{k}} w_{k}, \\
z_{k}=H_{\Theta_{k}} x_{k}+A_{k} \delta_{k}+D_{\Theta_{k}} v_{k},
\end{gathered}
$$

where $x_{k}$ and $z_{k}$ represent the system state and measurement, respectively. $\left\{\Theta_{k}\right\}$ is the state of Markov chain with finite state space $\{1, \cdots, M\}$ and transition probability matrix $P_{t}$ with its $(i, j)$ th element being $p_{i j}:=$ $P\left\{\Theta_{k+1}=j \mid \Theta_{k}=i\right\} . \pi_{j, k}:=P\left(\Theta_{k}=j\right)$ represents the $j$ th mode probability at instant $k . F_{\Theta_{k}}, G_{\Theta_{k}}, H_{\Theta_{k}}$, $A_{k}$ and $D_{\Theta_{k}}$ are known matrices with proper dimensions. $w_{k}$ and $v_{k}$ are zero-mean and white noises with covariances $Q_{k}$ and $R_{k}$, respectively, and independent of the initial state $x_{0}$ satisfying $E\left(x_{0} \mathbf{1}_{\left\{\Theta_{0}=i\right\}}\right)=\psi_{i, 0}$ and $E\left(x_{0} x_{0}^{T} \mathbf{1}_{\left\{\Theta_{0}=i\right\}}\right)=V_{i, 0}$. Here, $\left\{w_{k}\right\},\left\{v_{k}\right\}$ and $\left\{\Theta_{k}\right\}$ are independent mutually, and $\delta_{k}$ satisfies

$$
\left\{\begin{array}{l}
E\left\{\delta_{k} w_{l-1}^{T}\right\}=\mathrm{O} \\
E\left\{\delta_{k} v_{l}^{T}\right\}=\mathrm{O}
\end{array} \quad(\forall l \geq k) .\right.
$$

As shown in [14], $\delta_{k}$ represents a more general uncertainty (i.e. GUD): an arbitrary linear weighted sum of $f_{1 k}, f_{2 k}$ and $q_{k}$, where $f_{1 k}$ representing a class of UD with dynamic property is an linear time-varying function of $\bar{W}^{k-1}, \bar{V}^{k}$, and $\bar{\Delta}^{k-1}$ with $\bar{W}^{k-1}:=\left[w_{0}^{T}, \cdots, w_{k-1}^{T}\right]^{T}$, $\bar{V}^{k}:=\left[v_{1}^{T}, \cdots, v_{k}^{T}\right]^{T}$ and $\bar{\Delta}^{k-1}:=\left[\delta_{1}^{T}, \cdots, \delta_{k-1}^{T}\right]^{T} ; f_{2 k}$ representing deterministic UD is an arbitrary deterministic time-varying function; and $q_{k}$ representing random UD is white noise independent of $\bar{W}^{k-1}, \bar{V}^{k}$, and $\bar{\Delta}^{k-1}$.

Due to the presence of $\delta_{k}$, the routine of orthogonality principle for designing the LMMSE estimator will NOT work unless $\delta_{k}$ or its effect on estimation error covariance can be identified. In fact, such identification requires certain conditions which may be hardly satisfied:

- to identify the value of the GUD, the precondition is that the dimension of $\delta_{k}$ should be less than the rank of measurement matrix. Otherwise, the GUD has to be further constrained being piece-wise constant;

- to obtain the optimal filter gain $K=P_{x z} P_{z z}^{-1}$, we need to estimate the cross-covariance $P_{x z}$ between the predicted state and measurement and the innovation covariance $P_{z z}$. However, it is an intractable task due to the unknown relationship among $\delta_{k}$ and $\delta_{t}, w_{t-1}$ or $v_{t}$ for $t<k$.

It is highly demanded to develop a new filter with the looser design condition in pursuit of the best result in the worst situation.

Definition 1: (Definition of the UBF). A filter is called 
the UBF, i.e.,

$$
\begin{aligned}
& \left\{\hat{\xi}_{k+1 \mid k+1}, \Phi_{k+1 \mid k}^{*}, S_{k+1}^{*}, \Phi_{k+1 \mid k+1}^{*}\right\} \\
= & \operatorname{UBF}\left\{z_{k+1}, \hat{\xi}_{k \mid k}, \Phi_{k \mid k}^{*}\right\},
\end{aligned}
$$

if there exists a sequence of positive-definite matrices $\Phi_{k+1 \mid k}^{*}, S_{k+1}^{*}$ and $\Phi_{k+1 \mid k+1}^{*}$ that satisfy

$$
\begin{aligned}
\Phi_{k+1 \mid k}^{*} \geq \Phi_{k+1 \mid k} & :=E\left[\left(\xi_{k+1}-\hat{\xi}_{k+1 \mid k}\right)(\cdot)^{T}\right], \\
S_{k+1}^{*} \geq S_{k+1} & :=E\left[\left(z_{k+1}-\hat{z}_{k+1 \mid k}\right)(\cdot)^{T}\right], \\
\Phi_{k+1 \mid k+1}^{*} \geq \Phi_{k+1 \mid k+1} & :=E\left[\left(\xi_{k+1}-\hat{\xi}_{k+1 \mid k+1}\right)(\cdot)^{T}\right],
\end{aligned}
$$

where the geometry augmentation $\xi_{k}:=\operatorname{col}\left\{x_{k} \mathbf{1}_{\left\{\Theta_{k}=i\right\}}\right.$, $i=1, \cdots, M\} ; \hat{\xi}_{k \mid k}$ and $\hat{\xi}_{k+1 \mid k}$ are the estimate and prediction of $\xi_{k}$ given $Z_{1: k}:=\left\{z_{1}, \cdots, z_{k}\right\}$, respectively; $\hat{z}_{k+1 \mid k}$ is the measurement prediction; $\Phi_{k+1 \mid k}, S_{k+1}$ and $\Phi_{k+1 \mid k+1}$ are the covariances of the prediction error, innovation and estimate error, respectively; $\Phi_{k+1 \mid k}^{*}, S_{k+1}^{*}$ and $\Phi_{k+1 \mid k+1}^{*}$ are the corresponding upper bounds to be determined.

Our aim is to construct the upper bounds with one or more free parameters to guarantee the upper-boundness in (4)-(6) and further optimize parameters for the minimum upper bounds. As shown later, the adaptive estimation problem with GUDs is transformed into the online constrained parameter optimization.

Remark 2.1: The common idea of both the UBF and robust $H_{\infty}$ filters [16-19] is to obtain the best accuracy in the worst case. However, the considered estimation problem can't be solved by the $H_{\infty}$ filter because:

- the $H_{\infty}$ filter is designed to deal with norm-bounded parameter uncertainties, while the UBF are proposed for the statistically-equality-constrained GUDs which can be large at will. Even in the case that the GUD is random and its PDF is given, we treat it as the normbounded uncertainty under some confidence level, but the norm-unbounded case still exists with probability, which can not be dealt with by $H_{\infty}$ filter. Thus, the $H_{\infty}$ filter is not applicable to the GUD;

- parameter bounds should be known a priori in the $H_{\infty}$ filter design, while the UBF does not require to know the bound of the GUD.

Therefore, the UBF and the $H_{\infty}$ filter are distinct and possibly combined to cope with the coexistence of GUDs and norm-bounded parameter uncertainty in the future.

Remark 2.2: Though we have ever designed UBFs for the linear systems with GUDs in state dynamics [20] or the nonlinear systems with GUDs in sensors [14], there is no result suitable to the MJLSs due to the coexistence of Markov stochastic switching and GUDs. As shown in the following section, the filter derivation turns to designing the UBF for a more general system with stochastic parameters, determining the first two moments of stochastic parameters, and hence obtaining the UBF for MJLSs via parameter substitution.

\section{MUBF design for the MJLSs}

In the following, the related filter derivation will be presented in details. Firstly, a kind of more general polytopic systems with stochastic parameters and GUDs is considered and the corresponding UBF is designed. Then the MJLS with GUDs is transformed into a special case of the polytopic system and the recursive UBF is obtained, and further the optimal parameters are pursued for the minimum upper-bound filter (MUBF). Above consideration has two aspects advantages: one is the UBF for the general polytopic system can be obtained; the other is the filter recursion of the MJLSs is simplified since it can be gained by substituting directly the parameters into the UBF formulae of the polytopic system.

\subsection{UBF for polytopic systems with stochastic parame- ters}

Consider the following discrete-time polytopic system with stochastic parameters:

$$
\begin{gathered}
\xi_{k+1}=F_{k} \xi_{k}+G_{k} w_{k}, \\
z_{k}=H_{k} \xi_{k}+A_{k} \delta_{k}+D_{k} v_{k}
\end{gathered}
$$

with $\xi_{k}=\operatorname{col}\left\{\xi_{i, k}, i=1, \cdots, M\right\},\left[F_{k}\right]_{i, j}=\sum_{n=1}^{N} \alpha_{n, i j, k} F_{n, k}$, $\left[G_{k}\right]_{i, 1}=\sum_{n=1}^{N} \beta_{n, i, k} G_{n, k},\left[H_{k}\right]_{1, j}=\sum_{n=1}^{N} \varphi_{n, j, k} H_{n, k}$ and $D_{k}=\sum_{n=1}^{N} \mu_{n, k} D_{n, k} ; F_{n, k}, G_{n, k}, H_{n, k}$ and $D_{n, k}$ are the known basis matrices with proper dimensions; $\delta_{k}$ is the GUD satisfying (3); $A_{k}$ is the known disturbance coefficient matrix; $w_{k}$ and $v_{k}$ are zero-mean and white noises, independent of the initial state $\xi_{0}$; $\alpha_{n, i j, k}, \beta_{n, i, k}, \varphi_{n, j, k}$ and $\mu_{n, k}(j=1, \cdots, M, n=$ $1, \cdots, N)$ are stochastic parameters with $\bar{\alpha}_{n, i j, k}:=$ $E\left(\alpha_{n, i j, k}\right), C_{m n, i j p q, k}^{\alpha}:=E\left(\alpha_{m, i j, k}-\bar{\alpha}_{m, i j, k}\right)\left(\alpha_{n, p q, k}-\right.$ $\left.\bar{\alpha}_{n, p q, k}\right), \bar{\beta}_{n, i, k}:=E\left(\beta_{n, i, k}\right), C_{m n, i j, k}^{\beta}:=E\left(\beta_{m, i, k}-\right.$ $\left.\bar{\beta}_{m, i, k}\right)\left(\beta_{n, j, k}-\bar{\beta}_{n, j, k}\right), \bar{\varphi}_{n, j, k}:=E\left(\varphi_{n, j, k}\right), C_{m n, i j, k}^{\varphi}:=$ $E\left(\varphi_{m, i, k}-\bar{\varphi}_{m, i, k}\right)\left(\varphi_{n, j, k}-\bar{\varphi}_{n, j, k}\right), \bar{\mu}_{n, k}:=E\left(\mu_{n, k}\right)$, $C_{m n, k}^{\mu}:=E\left(\mu_{m, k}-\bar{\mu}_{m, k}\right)\left(\mu_{n, k}-\bar{\mu}_{n, k}\right)$.

Remark 3.1: In (7)-(8), the stochastic parameters (i.e. the random weights $\alpha_{k}, \beta_{k}, \varphi_{k}$ and $\mu_{k}$ ) represent the stochastic weights of polytopic matrices, that is, the parameter matrices randomly localize at a point within the polyhedron with its vertexes being the basis matrices $\left(F_{n, k}, G_{n, k}, H_{n, k}\right.$ and $\left.D_{n, k}\right)$. Due to the existence of stochastic parameters, the covariance of state prediction 
error will depend on current state, different from the existing UBFs in [14]. In other words, the coupling of stochastic parameters and current state should be considered in the derivation of the upper-bound covariances.

Theorem 1 (Existence of the UBF). If the following two conditions are satisfied

$$
\begin{gathered}
\Phi_{0 \mid 0}^{*} \geq \Phi_{0 \mid 0}, \\
S_{k+1}^{*} \geq S_{k+1},
\end{gathered}
$$

then there will exist UBFs with the following recursive upper-bound structure

$$
\begin{aligned}
\Phi_{k+1 \mid k}^{*}= & \Omega_{k+1}-\overline{\mathcal{F}}_{k} \Omega_{k} \overline{\mathcal{F}}_{k}^{T}+\overline{\mathcal{F}}_{k} \Phi_{k \mid k}^{*} \overline{\mathcal{F}}_{k}^{T} \\
S_{k+1}^{*}= & \overline{\mathcal{H}}_{k+1} \Phi_{k+1 \mid k}^{*} \overline{\mathcal{H}}_{k+1}^{T}+\varepsilon_{k+1} A_{k+1} \Sigma A_{k+1}^{T} \\
& +\tilde{\mathcal{R}}_{k+1}, \\
\Phi_{k+1 \mid k+1}^{*}= & \left(I-K_{k+1} H_{k+1}\right) \Phi_{k+1 \mid k}^{*}\left(I-K_{k+1} H_{k+1}\right)^{T} \\
& +K_{k+1} \tilde{\mathcal{R}}_{k+1} K_{k+1}^{T},
\end{aligned}
$$

and the filter recursion contains

$$
\begin{gathered}
\text { state prediction } \hat{\xi}_{k+1 \mid k}=\overline{\mathcal{F}}_{k} \hat{\xi}_{k \mid k}, \\
\text { measurement prediction } \hat{z}_{k+1 \mid k}=\overline{\mathcal{H}}_{k+1} \hat{\xi}_{k+1 \mid k}, \\
\text { filter residual } \gamma_{k+1}:=z_{k+1}-\hat{z}_{k+1 \mid k}, \\
\text { state estimation } \hat{\xi}_{k+1 \mid k+1}=\hat{\xi}_{k+1 \mid k}+K_{k+1} \gamma_{k+1},
\end{gathered}
$$

where $\varepsilon_{k+1}$ is a free nonnegative parameter to be estimated; $\Sigma$ is a positive-definite matrix; $\overline{\mathcal{F}}_{k}$ and $\overline{\mathcal{H}}_{k}$ represent $E\left(F_{k}\right)$ and $E\left(H_{k}\right)$ respectively; $\Omega_{k}:=E\left(\xi_{k} \xi_{k}^{T}\right)$ and $\tilde{\mathcal{R}}_{k}:=E\left(\tilde{H}_{k} \Omega_{k} \tilde{H}_{k}^{T}\right)+E\left(D_{k} R_{k} D_{k}^{T}\right)$ with $\tilde{H}_{k}:=H_{k}-\overline{\mathcal{H}}_{k} ;$ the filter gain $K_{k+1}$ is free here and will be optimized later according to $\Phi_{k+1 \mid k}^{*}$ and $S_{k+1}^{*}$.

\section{Proof: See Appendix A.}

Remark 3.2: In Theorem $1, \Sigma$ is user-defined and always positive-definite. In other words, such priori setting opens the door to introduce the application-specific expert knowledge. For example, if the user is particularly interested in attenuating the effect of uncertainty of the second element of $\delta_{k}$, then $\Sigma(2,2)$ should be chosen to be large relative to other elements of $\Sigma$. If the user knows a priori that the third element of $\delta_{k}$ is small, then $\Sigma(3,3)$ should be chosen to be small. As shown in the simulation, the derived filter is somewhat robust to the choice of $\Sigma$.

Remark 3.3: In Theorem 1, it is evident that the two existence conditions of the UBF are easy to be satisfied through choosing $\Phi_{0 \mid 0}^{*}=\Phi_{0 \mid 0}$ for the first condition and a large enough scalar $\varepsilon_{k+1}$ for the second one. The existence condition in (10) will be further explored to obtain the constraint on determining $\varepsilon_{k+1}$ later.

\subsection{MUBF for the MJLSs}

Denote $\left[F_{k}\right]_{i, j}=F_{j, k} \mathbf{1}_{\left\{\Theta_{k+1}=i \mid \Theta_{k}=j\right\}}, \quad\left[G_{k}\right]_{i, 1}=$ $\left(\sum_{j=1}^{M} G_{j, k} \mathbf{1}_{\left\{\Theta_{k}=j\right\}}\right) \mathbf{1}_{\left\{\Theta_{k+1}=i\right\}},\left[H_{k}\right]_{1, j}=H_{j, k}$ and $D_{k}=$ $\sum_{j=1}^{M} D_{j, k} \mathbf{1}_{\left\{\Theta_{k}=j\right\}}$ where the matrices $F_{j, k}, G_{j, k}, H_{j, k}$ and $D_{j, k}(i, j=1, \cdots, M)$ are related to the $j$ th mode. Obviously, the MJLS in (1)-(2) is a special case of the polytopic system in (7)-(8) with $\alpha_{n, i j, k}=\mathbf{1}_{\left\{\Theta_{k+1}=i \mid \Theta_{k}=j\right\}}$ if $n=j$ and 0 otherwise; $\beta_{j, i, k}=\mathbf{1}_{\left\{\Theta_{k}=j\right\}} \mathbf{1}_{\left\{\Theta_{k+1}=i\right\}}$; $\varphi_{n, j, k}=1$ if $n=j$ and 0 otherwise; $\mu_{i, k}=\mathbf{1}_{\left\{\Theta_{k}=i\right\}}$ for $n=1, \cdots, M$. Now, the original multi-mode complexity (i.e. $\left.\Theta_{k} \in\{1, \cdots, M\}\right)$ in (1)-(2) is transformed into the randomness of parameters in (7)-(8). The UBF filter for the MJLS in (1)-(2) is hence directly obtained through parameter substitution. In the following, we will specify $\tilde{R}_{k}, \Phi_{k+1 \mid k}$ and $\Omega_{i, k+1}$ for the UBF implementation.

Applying Theorem 1 to design the UBF for the MJLS, we have

$$
\tilde{R}_{k}=E\left(D_{k} R_{k} D_{k}^{T}\right)=\sum_{j=1}^{M} \pi_{j, k} D_{j, k} R_{k} D_{j, k}^{T},
$$

and the covariance of state prediction error

$$
\begin{aligned}
\Phi_{k+1 \mid k} & =\Omega_{k+1}-\Pi_{k+1 \mid k} \\
& =\operatorname{diag}\left\{\Omega_{i, k+1}, i=1, \cdots, M\right\}-\Pi_{k+1 \mid k}
\end{aligned}
$$

with $\Pi_{k \mid l}:=E\left(\hat{\xi}_{k \mid l} \hat{\xi}_{k \mid l}^{T}\right)$ and $\Omega_{k+1}=\operatorname{diag}\left\{\Omega_{i, k+1}, i=1\right.$, $\cdots, M\}$ satisfying

$$
\begin{aligned}
& \Omega_{i, k+1}=E\left(\xi_{i, k+1} \xi_{i, k+1}^{T}\right) \\
= & \sum_{j=1}^{M} p_{j i} F_{j, k} \Omega_{j, k} F_{j, k}^{T}+\sum_{j=1}^{M} p_{j i} \pi_{j, k} G_{j, k} Q_{k} G_{j, k}^{T} .
\end{aligned}
$$

Remark 3.4: A reviewer suggests an alternative way to derive $\Omega_{k+1}$ in (19) by letting $\Omega_{k}^{i}:=E\left[\xi_{k} \xi_{k}^{T} \mid \Theta_{k}=i\right] \pi_{i, k}$ and then obtaining $\Omega_{k+1}=\sum_{i=1}^{M} \Omega_{k+1}^{i}$ satisfying $\Omega_{k+1}^{i}=\bar{F}_{k}^{i} \sum_{j=1}^{M} \Omega_{k}^{i} p_{i j} \bar{F}_{k}^{i T}+\bar{G}_{k}^{i} Q_{k} \bar{G}_{k}^{i}{ }^{T} \pi_{i, k}$ with $\bar{F}_{k}^{i}:=F_{k}$ and $\bar{G}_{k}^{i}:=G_{k}$ for $\Theta_{k}=i$. It is a distinct derivation idea.

After obtaining the estimate $\hat{\xi}_{k+1 \mid k+1}$, we have

$$
\hat{x}_{k+1 \mid k+1}=\sum_{i=1}^{M} \hat{\xi}_{i, k+1 \mid k+1},
$$

where $\hat{\xi}_{i, k+1 \mid k+1}$ is the estimate of $x_{k+1} \mathbf{1}_{\Theta_{k+1}=i}$. 
Table 1

The MUBF Algorithm

Step 1: Initialization. Set initial values $\psi_{i, 0}$ and $V_{i, 0}$ for $i=1, \cdots, M$. Let $\hat{\xi}_{0 \mid 0}=\operatorname{col}\left\{\psi_{1,0}, \cdots, \psi_{M, 0}\right\}, \Omega_{0}=$ $\operatorname{diag}\left\{V_{1,0}, \cdots, V_{M, 0}\right\}$ and $\Pi_{0 \mid 0}=\hat{\xi}_{0 \mid 0} \hat{\xi}_{0 \mid 0}^{T}$.

Step 2: Prediction. Compute $\hat{\xi}_{k+1 \mid k}, \hat{z}_{k+1 \mid k}, \Omega_{i, k+1}$ and $\Phi_{k+1 \mid k}^{*}$ by (14), (15), (20) and (11), respectively.

Step 3: Filtering Residual. Compute $\gamma_{k+1}$ by (16).

Step 4: Parameter Optimization.

(a) Parameter Solving. Substitute related terms into (26), then solve for $\varepsilon_{k+1}$.

(b) Innovation covariance. Recompute $S_{k+1}^{*}$ by (12).

(c) Filter Gain. Compute the filter gain by (25).

Step 5: Estimation. Update the state $\hat{\xi}_{k+1 \mid k+1}$ by (17) and the upper-bound covariance $\Phi_{k+1 \mid k+1}^{*}$ by (13).

Step 6: Output. Compute $\hat{x}_{k+1 \mid k+1}$ by (21).

Theorem 2 (Parameter Optimization). If the initialization in (9) is satisfied and for any a $\varepsilon_{k+1}$ satisfying $S_{k+1} \leq\left. S_{k+1}^{*}\right|_{\varepsilon_{k+1}}$, there exists the MUBF, i.e.,

$$
\begin{gathered}
\Phi_{k+1 \mid k+1}\left|K_{k+1} \leq \Phi_{k+1 \mid k+1}^{*}\right| \varepsilon_{k+1}^{o p t}, K_{k+1}^{o p t} \\
\leq \Phi_{k+1 \mid k+1}^{*} \mid \varepsilon_{k+1}, K_{k+1} \\
S_{k+1} \leq S_{k+1}^{*}\left|\varepsilon_{k+1}^{o p t} \leq S_{k+1}^{*}\right|_{\varepsilon_{k+1}}
\end{gathered}
$$

and the MUBF has the following optimal parameters

$$
\begin{gathered}
\varepsilon_{k+1}^{o p t}=\min \left\{\varepsilon_{k+1} \mid \varepsilon_{k+1} \in \Lambda_{k+1}\right\} \\
K_{k+1}^{o p t}=\Phi_{k+1 \mid k}^{*} H_{k+1}^{T} S_{k+1}^{*-1}
\end{gathered}
$$

with $\Lambda_{k+1}:=\left\{\varepsilon_{k+1}\left|\varepsilon_{k+1} \geq 0, S_{k+1}^{*}\right|_{\varepsilon_{k+1}} \geq S_{k+1}\right\}$. Here, the parameter optimization in (24) can be solved as

$$
\varepsilon_{k+1}=\max \left(0, \frac{\sigma_{\max }\left(S_{k+1}^{0}\right)}{\sigma_{\min }\left(A_{k+1} \Sigma A_{k+1}^{T}\right)}\right),
$$

where $S_{k+1}^{0}=S_{k+1}-H_{k+1} \Phi_{k+1 \mid k}^{*} H_{k+1}^{T}-\tilde{R}_{k+1} ; \sigma_{\max }(\cdot)$ and $\sigma_{\min }(\cdot)$ represent the maximum and minimum eigenvalues, respectively.

\section{Proof: See Appendix B.}

In Theorem 2, $S_{k+1}$ is unknown and substituted by its unbiased estimate $\hat{S}_{k+1}=: \gamma_{k+1} \gamma_{k+1}^{T}$ in the filter. The implementation of the MUBF is given in Table 1.

Remark 3.5: The MUBF has multiple advantages:

- the measurement vector has at least one element while our MUBF has only one scalar parameter to be opti- mized. In other words, the MUBF will be always designable in principle;

- the estimation problem with GUD is transformed into the online scalar parameter optimization, and the resultant filter is adaptive instead of time-invariant in the $H_{\infty}$ filter design;

- by comparing the consistence between the measurement and its prediction, the introduced free parameter can be adjusted adaptively to compensate the modeling uncertainty and processing approximation in the filter design and hence the resultant filter is robust to modeling error and implementation approximation.

\subsection{Discussion of method extension}

In Theorem 2, the matrix $A_{k}$ is required full row-rank to insure the denominator $A_{k} \Sigma A_{k}^{T}$ in (26) have nonzero eigenvalue. However, $A_{k}$ may not be full row-rank in actual systems and thus the solvability condition of equality (26) will not be satisfied. For such case, the proposed MUBF still works via the following extension based on system decomposition.

First, introduce the row transformation $\Xi_{k}$ satisfying

$$
\Xi_{k} A_{k}=\left[\begin{array}{c}
A_{k}^{*} \\
\mathrm{O}
\end{array}\right]:=\bar{A}_{k},
$$

where $A_{k}^{*}$ is of full row-rank. Denoting $\bar{z}_{k}=\Xi_{k} z_{k}, \bar{H}_{k}=$ $\Xi_{k} H_{k}$ and $\bar{D}_{k}=\Xi_{k} D_{k}$ and multiplying $\Xi_{k}$ on both sides of (8) result in

$$
\bar{z}_{k}=\bar{H}_{k} \xi_{k}+\bar{A}_{k} \delta_{k}+\bar{D}_{k} v_{k}
$$

or its component expression

$$
\left\{\begin{array}{l}
z_{1, k}=H_{1, k} \xi_{k}+A_{k}^{*} \delta_{k}+D_{1, k} v_{k} \\
z_{2, k}=H_{2, k} \xi_{k}+D_{2, k} v_{k}
\end{array}\right.
$$

with $z_{1, k}$ and $z_{2, k}$ representing the $\delta$-coupled and $\delta$-free measurement components respectively.

From (29), we can obtain our resultant MUBF for $\delta$ coupled measurements based on the dynamic model in (7) and $z_{1, k}$ :

$$
\begin{aligned}
& \left\{\hat{\xi}_{1, k+1 \mid k+1}, \Phi_{1, k+1 \mid k}^{*}, S_{1, k+1}^{*}, \Phi_{1, k+1 \mid k+1}^{*}\right\} \\
= & \operatorname{MUBF}\left\{z_{1, k+1}, \hat{\xi}_{1, k \mid k}, \Phi_{1, k \mid k}^{*}\right\} .
\end{aligned}
$$

Meanwhile, the dynamic model in (7) along with $z_{2, k}$ of the $\delta$-free measurements is just the standard model of LMMSE estimator for MJLSs in [9]:

$$
\begin{aligned}
& \left\{\hat{\xi}_{2, k+1 \mid k+1}, \Phi_{2, k+1 \mid k}^{*}, S_{2, k+1}^{*}, \Phi_{2, k+1 \mid k+1}^{*}\right\} \\
= & \text { Costa_LMMSE }\left\{z_{2, k+1}, \hat{\xi}_{2, k \mid k}, \Phi_{2, k \mid k}^{*}\right\} .
\end{aligned}
$$


Remark 3.6: In fact, for the $\delta$-free measurement $z_{2, k}$, the Costa_LMMSE obtains the exact covariance under the LMMSE criterion, where it can be also treated as a special case of upper-bound covariances. In other word$\mathrm{s}$, the Costa_LMMSE can be regarded as the minimum upper-bound one among all possible UBFs.

In the scope of linear filter design, the final linear estimate is the weighted fusion of above two estimators:

$$
\hat{\xi}_{k \mid k}=C \hat{\xi}_{1, k \mid k}+(I-C) \hat{\xi}_{2, k \mid k}
$$

where $C$ is the parameter matrix to be optimized. The estimate error covariance is

$$
\begin{aligned}
& \Phi_{k \mid k}=E\left(C e_{1, k \mid k}+(I-C) e_{2, k \mid k}\right)(\cdot)^{T} \\
= & C \Phi_{1, k \mid k}^{*} C^{T}+(I-C) \Phi_{2, k \mid k}^{*}(I-C)^{T} \\
& +C \Phi_{12, k \mid k}(I-C)^{T}+\left(C \Phi_{12, k \mid k}(I-C)^{T}\right)^{T},
\end{aligned}
$$

where $e_{1, k \mid k}=\xi_{k}-\hat{\xi}_{1, k \mid k}, e_{2, k \mid k}=\xi_{k}-\hat{\xi}_{2, k \mid k}$, and $\Phi_{12, k \mid k}$, representing the cross-covariance between $e_{1, k \mid k}$ and $e_{2, k \mid k}$, has to be determined with same process and measurement noises. More seriously, it is unknown about the correlation between the GUD $\delta_{k}$ and past process/measurement noises. In other words, it is impossible to calculate $\Phi_{k \mid k}$ in (31). Hence, we optimize the trace of its upper-bound covariance $\Phi_{k \mid k}^{*}$ :

$$
\min _{C} \operatorname{trace}\left\{\Phi_{k \mid k}^{*}\right\} \quad \text { suject to } \quad \Phi_{k \mid k}^{*} \geq \Phi_{k \mid k}
$$

which can be solved via the Covariance Intersection Algorithm (CI) [21] with the following solution:

$$
C=\omega \Phi_{k \mid k}^{*} \Phi_{1, k \mid k}^{*-1}
$$

with

$$
\Phi_{k \mid k}^{*-1}=\omega \Phi_{1, k \mid k}^{*-1}+(1-\omega) \Phi_{2, k \mid k}^{*-1}
$$

For any a scalar parameter $\omega(0 \leq \omega \leq 1)$, it is proved that $\Phi_{k \mid k}^{*} \geq \Phi_{k \mid k}$ holds in (32).

Remark 3.7: As shown in (27), $A_{k}=0$ is equivalent to $\bar{A}_{k}=0$ in (28) or $A_{k}^{*}=0$ in (29). It means that the final state estimate equals to Costa_LMMSE $\{\cdot\}$, i.e., the LMMSE estimate in the case of $A_{k}=0$.

\section{Simulation}

Compared with the LMMSE estimator in [9], the proposed MUBF is testified by tracking a maneuvering target in $o-x y$ coordinate with GUDs. Here, two models are considered as follows: $F_{1}=I_{2} \otimes\left[\begin{array}{ll}1 & T \\ 0 & 1\end{array}\right]$,
$F_{2}=\left[\begin{array}{cccc}1 & \sin (\omega T) / \omega & 0 & -(1-\cos (\omega T)) / \omega \\ 0 & \cos (\omega T) & 0 & -\sin (\omega T) \\ 0 & (1-\cos (\omega T)) / \omega & 1 & \sin (\omega T) / \omega \\ 0 & \sin (\omega T) & 0 & \cos (\omega T)\end{array}\right]$, $G_{1}=G_{2}=I_{2} \otimes\left[\begin{array}{c}T^{2} / 2 \\ T\end{array}\right], H_{1}=H_{2}=I_{2} \otimes\left[\begin{array}{ll}1 & 0\end{array}\right]$, $D_{1}=D_{2}=I_{2}$ where $T=0.5 s, \omega=0.1257$. And $A_{k}=5 I_{2}, Q=0.01^{2} I_{2}$ and $R=0.5^{2} I_{2}$ in both models.

The GUDs are two random variables that both obey the uniform distribution in the interval $[-5,5]$. The maneuvering target is simulated with model 1 in the first 25 instants, model 2 in the middle 25 instants, and model 1 in the last 25 instants.

In the proposed MUBF and the LMMSE estimator in [9], the mode transition matrix equals $p_{11}=p_{22}=$ 0.98 , and the mode probabilities are $\pi_{1, k}=0.9, \pi_{2, k}=$ 0.1 for the first 25 instants, $\pi_{1, k}=0.1, \pi_{2, k}=0.9$ for the middle 25 instants, and $\pi_{1, k}=0.9, \pi_{2, k}=0.1$ for the last 25 instants, respectively. The initial state estimate is $\hat{x}_{0 \mid 0} \sim N\left(x_{0}, P_{0}\right)$ with the true initial state value being $x_{0}=(4,0.25,5,0.20)^{T}$ and $P_{0}=P_{0 \mid 0}^{*}=$ $\operatorname{diag}\{1,0.1,1,0.1\}$. Meanwhile, $\Sigma=10 I_{2}$.
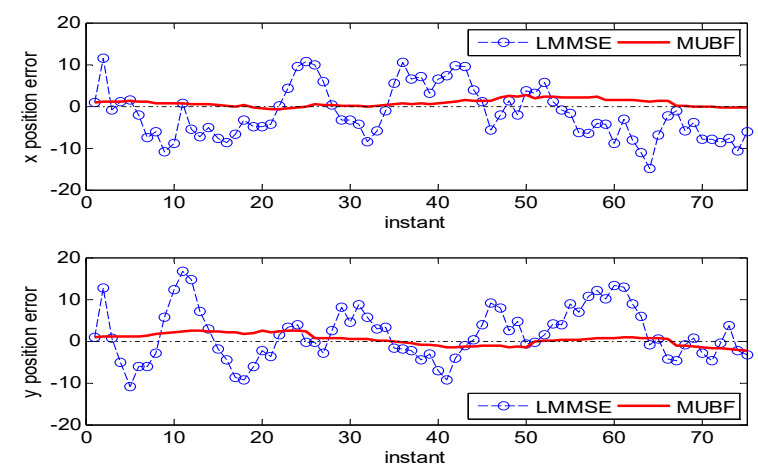

Fig. 1. Estimate errors in positions of the MUBF and LMMSE methods
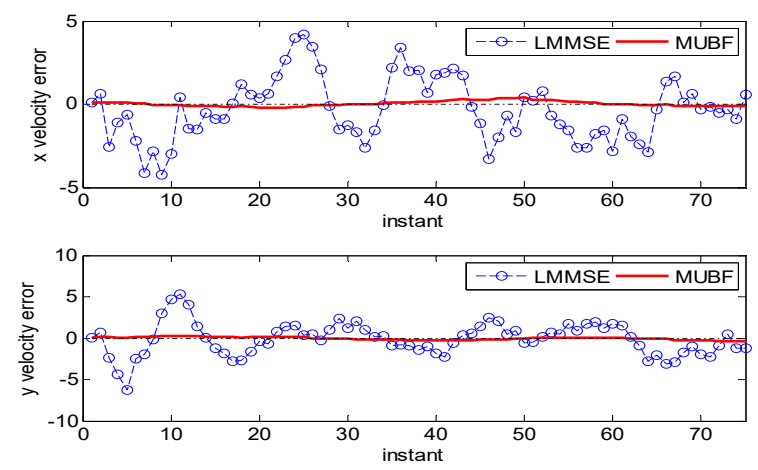

Fig. 2. Estimate errors in velocities of the MUBF and LMMSE methods 

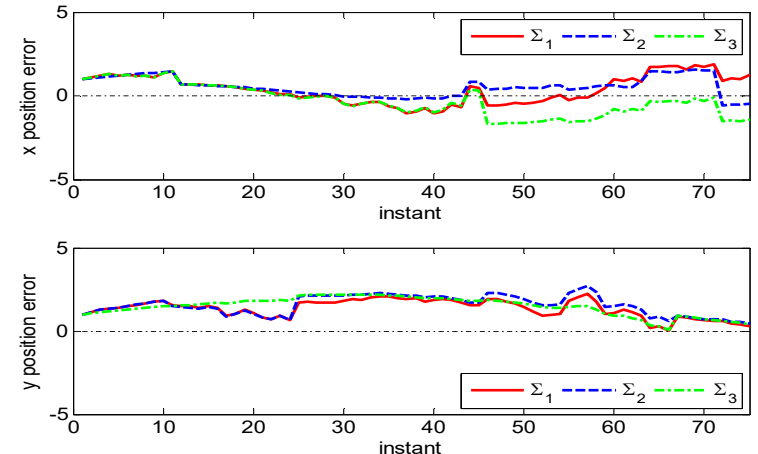

Fig. 3. Estimate errors in positions of the MUBF with different $\Sigma$
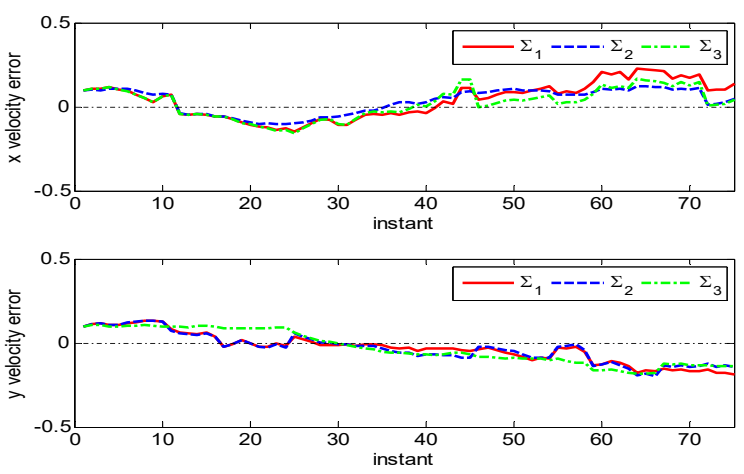

Fig. 4. Estimate errors in velocities of the MUBF with different $\Sigma$

In Figs. 1-2, the proposed MUBF obtains the less estimate errors in positions and velocities compared with the LMMSE in [9]. Meanwhile, as shown in Figs. 3-4, the MUBFs with different $\Sigma\left(\Sigma_{1}=\Sigma, \Sigma_{2}=\operatorname{diag}\{100,10\}\right.$ and $\left.\Sigma_{3}=\operatorname{diag}\{10,100\}\right)$ have the similar estimate errors, i.e., the proposed MUBF is robust to $\Sigma$.

\section{Conclusion}

A new MJLS with GUDs in measurements is considered. The UBF is proposed for the more general polytopic system with stochastic parameters and the existence conditions are given. Then, the MJLS with multimode complexity is transformed into the polytopic system via parameter substitution and thus the UBF can be used directly. Meanwhile, optimal parameters are derived so that the MUBF is obtained. Besides, the related method is extended from the full row-rank of $A_{k}$ to the general case.

\section{Acknowledgements}

This work is supported by China National Science Foundation (nos. 61374023, 61374159, 61135001) and the Doctorate Innovation Foundation of Northwestern Polytechnical University (no. CX201620).

\section{References}

[1] Y. Boers, and H. Driessen. A multiple model multiple hypothesis filter for Markovian switching systems. Automatica, 41:709-716, 2005.

[2] X. Li, and V. P. Jilkov. Survey of maneuvering target tracking. Part V: multiple-model methods. IEEE Trans. Aerosp. and Electron. Syst., 41(4):1255-1321, 2005.

[3] M. Liu, D. W.C. Ho, and P. Shi. Adaptive fault-tolerant compensation control for Markovian jump systems with mismatched external disturbance. Automatica, 58:5-14, 2015.

[4] H. Li, H. Gao, P. Shi, and X. Zhao. Fault-tolerant control of Markovian jump stochastic systems via the augmented sliding mode observer approach. Automatica, 50:1825-1834, 2014.

[5] J. Xiong, J. Lam, H. Gao, and D. W. C. Ho. On robust stabilization of Markovian jump systems with uncertain switching probabilities. Automatica, 41:897-903, 2005.

[6] L. A. Johnston, and V. Krishnamurthy. An improvement to the interacting multiple model (IMM) algorithm. IEEE Trans. Signal Process., 49(12):2909-2923, 2001.

[7] J. Lan, X. Li, and C. Mu. Best model augmentation for variable-structure multiple-model estimation. IEEE Trans. Aerosp. and Electron. Syst., 47(3):2008-2025, 2011.

[8] I. Bilik, and J. Tabrikian. Maneuvering target tracking in the presence of glint using the nonlinear gaussian mixture Kalman filter. IEEE Trans. Aerosp. and Electron. Syst., 46(1):246-262, 2010.

[9] O. L. V. Costa. Linear minimum mean square error estimation for discrete-time Markovian jump linear system. IEEE Trans. Automat. Control, 39(8):1685-1689, 1994.

[10] O. L. V. Costa. Stationary filter for linear minimum mean square wrror wstimation for discrete-time Markovian jump linear systems. IEEE Trans. Automat. Control, 47(8):13511356,2002 .

[11] M. H. Terra, J. Y. Ishihara, and A. P. Junior. Array algorithm for filtering of discrete-time Markovian jump linear systems. IEEE Trans. Automat. Control, 52(7):1293-1296, 2007.

[12] Y. Yang, Y. Liang, Q. Pan, Y. Qin, and F. Yang. Linear minimum-mean-square error estimation of Markovian jump linear systems with stochastic coefficient matrices. IET Control Theory Appl., 8(12):1112-1126, 2014.

[13] Y. Yang, Y. Liang, F. Yang, Y. Qin, and Q. Pan. Linear minimum-mean-square error estimation of Markovian jump linear systems with randomly delayed measurements. IET Signal Process., 8(6):658-667, 2014.

[14] Y. Qin, Y. Liang, Y. Yang, Z. Wang, and F. Yang. Adaptive filter of non-linear systems with generalized unknown disturbances. IET Radar Sonar Navig., 4(8):307-317, 2014.

[15] M. Greco, F. Gini, and A. Farina. Radar detection and classification of jamming signals belonging to a cone class. IEEE Trans. Signal Process., 56(5):1984-1993, 2008.

[16] L. Zhang. $H_{\infty}$ estimation for discrete-time piecewise homogeneous Markov jump linear systems. Automatica, 45: 2570-2576, 2009

[17] X. Zhao, and Q. Zeng. New robust delay-dependent stability and $H_{\infty}$ analysis for uncertain Markovian jump systems with time-varying delays. J. Franklin Institute, 347:863-874, 2010.

[18] J. Xiong, and J. Lam. Fixed-order robust $H_{\infty}$ filter design for Markovian jump systems with uncertain switching probabilities. IEEE Trans. Signal Process., 54(4):1421-1430, 2006. 
[19] L. Zhang, and E. Boukas. Mode-dependent $H_{\infty}$ filtering for discrete-time Markovian jump linear systems with partly unknown transition probabilities. Automatica, 45:1462-1467, 2009.

[20] Y. Liang, D. Zhou, L. Zhang, and Q. Pan. Adaptive filtering for stochastic systems with generalized disturbance inputs. IEEE Signal Process. Lett., 15:645-648, 2008.

[21] L. Chen, P. O. Arambel, and R. K. Mehra. Estimation under unknown correlation: covariance intersection revisited. IEEE Trans. Automat. Control, 47(11):1879-1882, 2002.

\section{A Appendix A: The Proof of Theorem 1}

First, we demonstrate that the upper-bound $S_{k+1}^{*}$ satisfying (12) guarantees $S_{k+1}^{*} \geq S_{k+1}$ in (5). Denote $\Upsilon_{k \mid l}:=E\left(\xi_{k}-\hat{\xi}_{k \mid l}\right)\left(z_{k}-\hat{z}_{k \mid l}\right)^{T}$ and $\tilde{\varphi}_{i, m, k}=\varphi_{i, m, k}-$ $\bar{\varphi}_{i, m, k}$. In (14) and (15), we have

$$
\begin{aligned}
\hat{\xi}_{k+1 \mid k} & =E\left(F_{k} \xi_{k} \mid Z_{1: k}\right)+E\left(G_{k} w_{k} \mid Z_{1: k}\right) \\
& =\overline{\mathcal{F}}_{k} \hat{\xi}_{k \mid k}, \\
\hat{z}_{k+1 \mid k}= & E\left(H_{k+1} \xi_{k+1}+A_{k+1} \delta_{k+1}+D_{k+1} v_{k+1} \mid Z_{1: k}\right) \\
= & \overline{\mathcal{H}}_{k+1} \hat{\xi}_{k+1 \mid k} .
\end{aligned}
$$

In above derivation, (A.1) holds since $E\left(G_{k} w_{k} \mid Z_{1: k}\right)=$ $O$. (A.2) holds since $E\left(D_{k+1} v_{k+1} \mid Z_{1: k}\right)=O$ and the best approximation of $E\left(A_{k} \delta_{k+1} \mid Z_{1: k}\right)$ is zero as $\delta_{k+1}$ is the unknown term. Then, $\gamma_{k+1}=H_{k+1} \xi_{k+1}-\overline{\mathcal{H}}_{k+1} \hat{\xi}_{k+1 \mid k}+$ $A_{k+1} \delta_{k+1}+D_{k+1} v_{k+1}$. By the fact that $v_{k+1}$ is independent of $\xi_{k+1}, \hat{\xi}_{k+1 \mid k}$ and $\delta_{k+1}$, we have

$$
\begin{aligned}
S_{k+1}= & E\left(H_{k+1} \xi_{k+1}-\overline{\mathcal{H}}_{k+1} \hat{\xi}_{k+1 \mid k}+A_{k+1} \delta_{k+1}\right)(\cdot)^{T} \\
& +E\left(D_{k+1} v_{k+1} v_{k+1}^{T} D_{k+1}^{T}\right) .
\end{aligned}
$$

As shown in (A.3), the presence of $\delta_{k+1}$ represents the uncertainty. Thus

$$
\begin{aligned}
\mathrm{S}_{k+1} \geq & E\left(H_{k+1} \xi_{k+1}-\overline{\mathcal{H}}_{k+1} \hat{\xi}_{k+1 \mid k}\right)(\cdot)^{T} \\
& +E\left(D_{k+1} v_{k+1} v_{k+1}^{T} D_{k+1}^{T}\right) \\
= & E\left(\left(H_{k+1}-\overline{\mathcal{H}}_{k+1}\right) \xi_{k+1}+\overline{\mathcal{H}}_{k+1}\left(\xi_{k+1}-\hat{\xi}_{k+1 \mid k}\right)\right)(\cdot)^{T} \\
& +E\left(D_{k+1} R_{k+1} D_{k+1}^{T}\right) \\
= & \overline{\mathcal{H}}_{k+1} \Phi_{k+1 \mid k} \overline{\mathcal{H}}_{k+1}^{T}+\tilde{\mathcal{R}}_{k+1}
\end{aligned}
$$

where

$$
\begin{aligned}
\Phi_{k+1 \mid k} & =E\left(\xi_{k+1} \xi_{k+1}^{T}\right)-E\left(\hat{\xi}_{k+1 \mid k} \hat{\xi}_{k+1 \mid k}^{T}\right) \\
& =\Omega_{k+1}-\Pi_{k+1 \mid k}
\end{aligned}
$$

with

$$
\begin{aligned}
\Pi_{k+1 \mid k} & =E\left(\overline{\mathcal{F}}_{k} \hat{\xi}_{k \mid k} \hat{\xi}_{k \mid k}^{T} \overline{\mathcal{F}}_{k}^{T}\right)=\overline{\mathcal{F}}_{k} \Pi_{k \mid k} \overline{\mathcal{F}}_{k}^{T}, \\
\Pi_{k+1 \mid k+1} & =E\left(\hat{\xi}_{k+1 \mid k} \xi_{k+1 \mid k}^{T}\right)+\left(\Upsilon_{k+1 \mid k} S_{k+1}^{-1}\right) S_{k+1}(\cdot)^{T} \\
& =\Pi_{k+1 \mid k}+\Upsilon_{k+1 \mid k} S_{k+1}^{-1} \Upsilon_{k+1 \mid k}^{T},
\end{aligned}
$$

$$
\begin{aligned}
\Upsilon_{k+1 \mid k} & =E\left(\xi_{k+1} \xi_{k+1}^{T} H_{k+1}^{T}\right)-E\left(\hat{\xi}_{k+1 \mid k} \hat{\xi}_{k+1 \mid k}^{T} \overline{\mathcal{H}}_{k+1}^{T}\right) \\
& =\Phi_{k+1 \mid k} \overline{\mathcal{H}}_{k+1}^{T} .
\end{aligned}
$$

Moreover, $\Omega_{i j, k+1}:=E\left(\xi_{i, k+1} \xi_{j, k+1}^{T}\right)$, as the $(i, j)$ th subblock of $\Omega_{k+1}$, can be calculated as (A.9) (at the top of next Page). Then, both terms in $\tilde{\mathcal{R}}_{k+1}$ are obtained as (A.10) and (A.11), respectively (at the top of next Page).

Thus, from (A.4), it is obvious that (5) can be easily obtained given $S_{k+1}^{*}$ with structure in (12). Then, we need to proof that (4) and (6) are satisfied when given (11) and (13), respectively.

From above derivation, it is easy to know

$$
\begin{aligned}
& \Phi_{k+1 \mid k}=\Omega_{k+1}-\overline{\mathcal{F}}_{k} \Omega_{k} \overline{\mathcal{F}}_{k}^{T}+\overline{\mathcal{F}}_{k} \Phi_{k \mid k} \overline{\mathcal{F}}_{k}^{T}, \\
& \Phi_{k+1 \mid k+1}=\Omega_{k+1}-\Pi_{k+1 \mid k+1} \\
&= \Phi_{k+1 \mid k}-\Upsilon_{k+1 \mid k} S_{k+1}^{-1} \Upsilon_{k+1 \mid k}^{T} \\
&=\left(I-K_{k+1} H_{k+1}\right) \Phi_{k+1 \mid k}\left(I-K_{k+1} H_{k+1}\right)^{T} \\
&+K_{k+1} \tilde{\mathcal{R}}_{k+1} K_{k+1}^{T} .
\end{aligned}
$$

Considering the first condition in (9) of Theorem 1 and assuming $\Phi_{k \mid k}^{*} \geq \Phi_{k \mid k}$, then using mathematical induction and (A.12), we have

$$
\Phi_{k+1 \mid k}^{*}-\Phi_{k+1 \mid k}=\overline{\mathcal{F}}_{k}\left(\Phi_{k \mid k}^{*}-\Phi_{k \mid k}\right) \overline{\mathcal{F}}_{k}^{T}
$$

As $\overline{\mathcal{F}}_{k}$ is a full-rank square matrix, we can obtain

$$
\Phi_{k+1 \mid k}^{*}-\Phi_{k+1 \mid k} \geq 0 .
$$

Thus, from (A.12) and (A.15), (4) is satisfied given (11).

Finally, putting (4) into (13) yields

$$
\begin{aligned}
\Phi_{k+1 \mid k+1}^{*}= & \left(I-K_{k+1} H_{k+1}\right) \Phi_{k+1 \mid k}^{*}\left(I-K_{k+1} H_{k+1}\right)^{T} \\
& +K_{k+1} \tilde{\mathcal{R}}_{k+1} K_{k+1}^{T} \\
\geq & \left(I-K_{k+1} H_{k+1}\right) \Phi_{k+1 \mid k}\left(I-K_{k+1} H_{k+1}\right)^{T} \\
& +K_{k+1} \tilde{\mathcal{R}}_{k+1} K_{k+1}^{T} \\
= & \Phi_{k+1 \mid k+1} .
\end{aligned}
$$

According to (A.16), (6) holds given (13).

\section{B Appendix B: The Proof of Theorem 2}

According to Theorem 2, the set $\left\{\varepsilon_{k+1} \mid S_{k+1}^{*} \geq S_{k+1}\right\}$ will not be empty if an UBF exists. In the case that $S_{k+1}^{*}\left|\varepsilon_{k+1,1} \leq S_{k+1}^{*}\right| \varepsilon_{k+1,2}$ for $\varepsilon_{k+1,1} \leq \varepsilon_{k+1,2}, \varepsilon_{k+1,2} \in$ $\Lambda_{k+1}$ holds if $\varepsilon_{k+1,1} \in \Lambda_{k+1}$. Thus

$$
\begin{aligned}
\Lambda_{k+1} & =\left\{\varepsilon_{k+1}\left|\varepsilon_{k+1} \geq 0, S_{k+1}^{*}\right| \varepsilon_{k+1} \geq S_{k+1}\right\} \\
& =\left\{\varepsilon_{k+1} \mid \varepsilon_{k+1} \geq 0\right\} \cap\left\{\varepsilon_{k+1}\left|S_{k+1}^{*}\right| \varepsilon_{k+1} \geq S_{k+1}\right\}
\end{aligned}
$$




$$
\begin{aligned}
& \Omega_{i j, k+1}=E\left(\left(\left[\sum_{n=1}^{N} \alpha_{n, i 1, k} F_{n, k} \cdots \sum_{n=1}^{N} \alpha_{n, i M, k} F_{n, k}\right]\right) \xi_{k} \xi_{k}^{T}(\cdot)^{T}\right)+E\left(\left(\sum_{n=1}^{N} \beta_{n, i, k} G_{n} w_{k}\right)(\cdot)^{T}\right) \\
= & \sum_{l=1}^{N} \sum_{q=1}^{N} \sum_{m=1}^{N} \sum_{n=1}^{N} E\left(\alpha_{n, i q, k} \alpha_{m, j l, k}\right) F_{n, k} \Omega_{q l, k+1} F_{m, k}^{T}+\sum_{n=1}^{N} \sum_{m=1}^{N} E\left(\beta_{n, i, k} \beta_{m, j, k}\right) G_{n, k} Q_{k} G_{m, k}^{T} \\
= & \sum_{l=1}^{N} \sum_{q=1}^{N} \sum_{m=1}^{N} \sum_{n=1}^{N}\left(\bar{\alpha}_{n, i q, k} \bar{\alpha}_{m, j l, k}+C_{n m, i q j l, k}^{\alpha}\right) F_{n, k} \Omega_{q l, k+1} F_{m, k}^{T}+\sum_{n=1}^{N} \sum_{m=1}^{N}\left(\bar{\beta}_{n, i, k} \bar{\beta}_{m, j, k}+C_{n m, i j, k}^{\beta}\right) G_{n, k} Q_{k} G_{m, k}^{T} . \\
& E\left(\tilde{H}_{k+1} \Omega_{k+1} \tilde{H}_{k+1}^{T}\right)=E\left(\left(\left[\sum_{l=1}^{N} \tilde{\varphi}_{l, 1, k+1} H_{l, k+1} \cdots \sum_{l=1}^{N} \tilde{\varphi}_{l, M, k} H_{l, k+1}\right]\right) \Omega_{k+1}(\cdot)\right) \\
= & \sum_{n=1}^{N} \sum_{m=1}^{N} \sum_{l=1}^{N} \sum_{q=1}^{N} E\left(\tilde{\varphi}_{l, m, k} \tilde{\varphi}_{q, n, k}\right) H_{l, k} \Omega_{m n, k+1} H_{q, k}^{T}=\sum_{n=1}^{N} \sum_{m=1}^{N} \sum_{l=1}^{N} \sum_{q=1}^{N} C_{l q, m n, k}^{\varphi} H_{l, k} \Omega_{m n, k+1} H_{q, k}^{T}, \\
& E\left(D_{k+1} R_{k+1} D_{k+1}^{T}\right)=E\left(\left(\sum_{i=1}^{N} \mu_{i, k+1} D_{i, k}\right) R_{k+1}(\cdot)^{T}\right)=\sum_{j=1}^{N} \sum_{i=1}^{N}\left(\bar{\mu}_{i, k+1} \bar{\mu}_{j, k+1}+C_{i j, k+1}^{\mu}\right) D_{i, k} R_{k+1} D_{j, k}^{T} .
\end{aligned}
$$

is not empty. Hence there exists $\varepsilon_{k+1}^{o p t}=\min \left\{\varepsilon_{k+1} \mid \varepsilon_{k+1} \in\right.$ $\left.\Lambda_{k+1}\right\}$.

Then, it is only necessary to testify that $\varepsilon_{k+1}^{o p t}$ and $K_{k+1}^{\text {opt }}$ guarantee (22)-(23). It is easy to know $\varepsilon_{k+1} \geq$ $\varepsilon_{k+1}^{o p t}$ for any $\varepsilon_{k+1} \in \Lambda_{k+1}$. Since $\Phi_{k+1 \mid k+1}^{*-1}=\Phi_{k+1 \mid k}^{*-1}+$ $H_{k+1}^{T}\left(\varepsilon_{k+1} A_{k+1} \Sigma A_{k+1}^{T}+\tilde{R}_{k+1}\right) H_{k+1}$ with $\tilde{R}_{k+1}$ in (18), $\left.\Phi_{k+1 \mid k}^{*-1}\right|_{\varepsilon_{k+1}}-\left.\Phi_{k+1 \mid k}^{*-1}\right|_{\varepsilon_{k+1}^{o p t}}=O$ and $\varepsilon_{k+1} \geq \varepsilon_{k+1}^{o p t} \geq 0$, thus we can obtain

$$
\begin{gathered}
\quad \Delta S_{k+1}=\left.S_{k+1}^{*}\right|_{\varepsilon_{k+1}}-\left.S_{k+1}^{*}\right|_{\varepsilon_{k+1}^{o p t}} \\
=\left(H_{k+1} \Phi_{k+1 \mid k}^{*} H_{k+1}^{T}+\varepsilon_{k+1} A_{k+1} \Sigma A_{k+1}^{T}+\tilde{R}_{k+1}\right) \\
\quad-\left(H_{k+1} \Phi_{k+1 \mid k}^{*} H_{k+1}^{T}+\varepsilon_{k+1}^{o p t} A_{k+1} \Sigma A_{k+1}^{T}+\tilde{R}_{k+1}\right) \\
=\left(\varepsilon_{k+1}-\varepsilon_{k+1}^{o p t}\right) A_{k+1} \Sigma A_{k+1}^{T} \geq 0, \\
\Delta \Phi_{k+1 \mid k+1}^{-1}=\left.\Phi_{k+1 \mid k+1}^{*-1}\right|_{\varepsilon_{k+1}, K_{k+1}}-\Phi_{k+1 \mid k+1}^{*-1}||_{\varepsilon_{k+1}^{o p t}, K_{k+1}} \\
=\left.\Phi_{k+1 \mid k}^{*-1}\right|_{\varepsilon_{k+1}}-\left.\Phi_{k+1 \mid k}^{*-1}\right|_{\varepsilon_{k+1}^{o p t}} \\
\quad+H_{k+1}^{T}\left(\varepsilon_{k+1} A_{k+1} \Sigma A_{k+1}^{T}+\tilde{R}_{k+1}\right)^{-1} H_{k+1} \\
\quad-H_{k+1}^{T}\left(\varepsilon_{k+1}^{o p t} A_{k+1} \Sigma A_{k+1}^{T}+\tilde{R}_{k+1}\right)^{-1} H_{k+1} \\
\leq 0 .
\end{gathered}
$$

From (B.2), we have

$$
\left.\Phi_{k+1 \mid k+1}^{*}\right|_{\varepsilon_{k+1}, K_{k+1}} \geq\left.\Phi_{k+1 \mid k+1}^{*}\right|_{\varepsilon_{k+1}^{o p t}, K_{k+1}} .
$$

Through (12) and $\tilde{R}_{k+1}>0, S_{k+1}^{*} \geq \tilde{R}_{k+1}>0$ exists, so the symmetric and positive-definite matrix $S_{k+1}^{*} \mid \varepsilon_{k+1}^{o p t}$ can be represented by $G_{k+1} G_{k+1}^{T}$, where $G_{k+1}$ is full rank. Suppose $K_{k+1}^{o p t}$ exists, and then its existence needs to be testified. After simple derivation, we have

$$
\begin{aligned}
& \left.\Phi_{k+1 \mid k+1}^{*}\right|_{\varepsilon_{k+1}^{o p t}, K_{k+1}}=\Phi_{k+1 \mid k}^{*} \mid \varepsilon_{k+1}^{o p t}-B_{k+1} B_{k+1}^{T} \\
& +\left(K_{k+1} G_{k+1}-B_{k+1}\right)\left(K_{k+1} G_{k+1}-B_{k+1}\right)^{T}
\end{aligned}
$$

with $B_{k+1}=\left.\Phi_{k+1 \mid k}^{*}\right|_{\varepsilon_{k+1}^{o p t}} H_{k+1}^{T} G_{k+1}^{-T}$. Since $\left(K_{k+1} G_{k+1}-\right.$ $\left.B_{k+1}\right)(\cdot)^{T} \geq 0$, we have $\Phi_{k+1 \mid k+1}^{*}=\left.\Phi_{k+1 \mid k}^{*}\right|_{\varepsilon_{k+1}^{o p t}}-$ $B_{k+1} B_{k+1}^{T}$ if and only if $K_{k+1}^{o p t}=B_{k+1} G_{k+1}^{-1}=$ $\left.\Phi_{k+1 \mid k}^{*}\right|_{\varepsilon_{k+1}^{o p t}} H_{k+1}^{T} S_{k+1}^{*-1}$. So, there exists

$$
\begin{aligned}
\left.\Phi_{k+1 \mid k+1}^{*}\right|_{\varepsilon_{k+1}^{o p t}, K_{k+1}} & \geq \Phi_{k+1 \mid k}^{*} \mid \varepsilon_{k+1}^{o p t}-B_{k+1} B_{k+1}^{T} \\
& =\Phi_{k+1 \mid k+1}^{*} \mid \varepsilon_{k+1}^{o p t}, K_{k+1}^{o p t} .
\end{aligned}
$$

Thus, an MUBF exists and $\varepsilon_{k+1}^{o p t} \in \Lambda_{k+1}$, i.e. (4)-(6) are obtained and (22)-(23) are further obtained based on (4)-(6), (B.1), (B.3) and (B.5).

From (5), (12) and (18), we have

$$
\begin{aligned}
S_{k+1}^{*} & =H_{k+1} \Phi_{k+1 \mid k}^{*} H_{k+1}^{T}+\varepsilon_{k+1} A_{k+1} \Sigma A_{k+1}^{T}+\tilde{R}_{k+1} \\
& \geq S_{k+1} .
\end{aligned}
$$

That is

$$
\varepsilon_{k+1} A_{k+1} \Sigma A_{k+1}^{T} \geq S_{k+1}-H_{k+1} \Phi_{k+1 \mid k}^{*} H_{k+1}^{T}-\tilde{R}_{k+1} .
$$

Hence, the optimal parameter in (26) can be obtained as the solution of $\varepsilon_{k+1}$. 